\section{'Gin in a tin' sugar content exposed in survey}

A new product survey conducted by Action on Sugar has shown that popular 'ready to drink' pre-mixed spirits and cocktails sold in supermarkets are extremely high in hidden sugar and calories.

To mark Sugar Awareness Week (20-26 January 2020), Action on Sugar called on the new government to ensure that all alcoholic drinks are included in vital public health policies, and said that major UK retailers should be forced to reformulate their pre-mixed alcoholic drinks to the agreed criterion set by government in the Soft Drinks Industry Levy (SDIL) or pay the fine.

Some of the beverages surveyed contained nine teaspoons of sugar in just $250 \mathrm{ml}$ (as well as alcohol) and nine out of ten pre-mixed spirits did not have on-pack sugar information.

WKD Blue contained $59 \mathrm{~g}$ of sugar in a $700 \mathrm{ml}$ bottle - the equivalent of eating over four iced doughnuts in one sitting. Classic Combinations Pink Gin and Tonic contained $27 \mathrm{~g}$ of sugar in a $250 \mathrm{ml}$ can, the same sugar content as Coca Cola.

Katharine Jenner, Campaign Director at Action on Sugar, based at Queen Mary University of London, said: “'Gin in a tin" has become a cultural phenomenon with these types of drinks often consumed "on the go" and without a moment's consideration to how much sugar and alcohol goes into making them. Even if you did want to know, you can't make a healthy choice as only one in ten of the products surveyed had enough information available. If consumers knew how much sugar was really in these drinks, would they still happily choose to drink their way to tooth decay, obesity and type 2 diabetes?'

\section{Cancer patient starts petition for free dental care}

Michele Solak of Manchester has started a petition campaigning to get free dental care for cancer patients and at the time of writing has already received over 140,000 signatures in under two weeks.

Michele was diagnosed with triple negative breast cancer in 2016. Whilst undergoing treatment, she had no idea that dental treatment didn't fall under the prescription exemption for cancer patients. She started a Facebook group for other people who had been diagnosed specifically with triple negative breast cancer and noticed a link between dental problems and cancer.

Many people, who seemingly had no dental problems prior to undergoing cancer treatment, found they were suffering multiple problems with their teeth, such as crumbling, teeth falling out and other painful symptoms. Michele has spoken to women who were having to undergo multiple root canal treatments, denture fixtures, crown fittings and more. The cost of this meant people were having to pay up to $£ 4,000$ in some cases at a time when they were already undergoing a lot of financial and emotional stress.

Michele was spurred to start the petition after the general election, as she noticed the government putting so much focus on the NHS.

Prevent Breast Cancer founder, Chairman and Consultant Breast Surgeon, Mr Lester Barr said: 'Breast cancer patients can run into problems with their teeth and need dental care as part of their overall treatment. Just like prescription charges, it makes sense to provide this free of charge for patients receiving chemotherapy or bisphosphonates for cancer'.

To see Michele's petition, visit https://bit.ly/2OuBLdi.

\title{
'Check before you tick' campaign launched
}

From 20 January 2020 new activity from NHS England and NHS Improvement, and NHS Business Services Authority (NHSBSA), encourages dental teams across England to support the NHS 'Check before you tick' campaign to help patients understand their eligibility for free or reduced cost NHS dental treatment.

The campaign is an extension of ongoing 'Check before you tick' prescriptions activity which launched for the first time in September 2018, helping patients to understand if they are entitled to receive free prescriptions and how to claim correctly to avoid unnecessary penalty charges.

The NHS makes checks on free and reduced cost dental claims each month; therefore, the campaign is important in helping to support dental teams to raise awareness of the need for people to check their entitlement using the NHS online eligibility checker. The eligibility checker can also let patients know about other means of support available to help them reduce the costs of dental treatment, such as the NHS Low Income Scheme.

A briefing, along with a toolkit of posters, counter cards and information booklets have been produced and sent to dental practices for use all year round to help remind patients to always 'check before you tick', using the NHS online eligibility checker. The materials and further digital resources are also available to download via the Campaign Resource Centre (https://campaignresources.phe.gov.uk/resources/ campaigns/100-check-before-you-tick-).

The campaign focuses primarily on patients making benefitrelated claims who, according to audience research, can mistakenly assume they're automatically entitled to free dental treatment and may therefore need more help understanding their eligibility. There is also low awareness that the NHS makes checks on claims for free dental treatment and can issue a penalty charge of $£ 100$ in addition to the cost of treatment to those who have claimed incorrectly.

Dental teams are being encouraged to display the materials prominently, ensure staff are familiar with the eligibility criteria, and highlight to patients to check their entitlement before completing the patient declaration form.

Information about eligibility is also available to download from https://www.nhsbsa.nhs.uk/dont-get-caught-out-penalty-charges/ check-you-tick in a number of alternative formats, including large print, easy read and BSL.

The online eligibility checker can be accessed at https://www. nhsbsa.nhs.uk/dont-get-caught-out-penalty-charges/check-you-tick. 